\title{
Transglutaminase 2: A Novel Autoantigen in Canine Idiopathic Central Nervous System Inflammatory Diseases
}

\author{
Miho TANAKA ${ }^{1)}$, Akiko INOUE ${ }^{1)}$, Kei YAMAMOTO ${ }^{1)}$, Satoshi TAMAHARA ${ }^{1)}$ and Naoaki MATSUKI ${ }^{1) *}$ \\ ${ }^{1)}$ Department of Veterinary Clinical Pathobiology, Graduate School of Agricultural and Life Sciences, The University of Tokyo, \\ 1-1-1 Yayoi, Bunkyo-ku, Tokyo 113-8657, Japan
}

(Received 9 November 2011/Accepted 25 December 2011/Published online in J-STAGE 10 January 2012)

ABSTRACT. Necrotizing meningoencephalitis (NME), necrotizing leukoencephalitis (NLE) and granulomatous meningoencephalomyelitis (GME) are common idiopathic inflammatory central nervous system (CNS) diseases with unknown etiology in dogs. We previously showed that IgG autoantibodies in the cerebrospinal fluid (CSF) of NME cases reacted to unknown brain proteins as well as to glial fibrillary acidic protein (GFAP). In the present report, we evaluated the autoantibodies against transglutaminase2 (TG2) in the canine CNS diseases. CSF samples obtained from dogs with NME ( $n=19)$, NLE $(n=7)$, GME $(n=11)$ and miscellaneous CNS diseases $(\mathrm{n}=12)$ were subjected. CSFs from 20 healthy dogs were used as controls. Indirect fluorescent antibody test on the canine cerebrum revealed astrocyte-binding IgG in the CSF of NME. After absorption of the CSF with bovine GFAP, the CSF still possessed the reactivity to astrocytes. Double-color staining showed clear colocalization of the autoantibodies and anti-human TG2 rabbit polyclonal IgG. An immunoblot assay against human recombinant TG2 revealed anti-TG2 IgG in the CSF from dogs with NME, NLE and GME. The CSF of canine idiopathic encephalitis cases, notably of NME, tended to show high ELISA OD values against human recombinant TG2 compared to healthy controls. The presence of anti-TG2 autoantibodies in the CSF may contribute to the elucidation of the etiology of canine NME, NLE and GME.

KEY WORDS: autoantibody, canine, cerebrospinal fluid, encephalitis, transglutaminase.

doi: 10.1292/jvms.11-0507; J. Vet. Med. Sci. 74(6): 733-737, 2012

Necrotizing meningoencephalitis (NME), necrotizing leukoencephalitis (NLE) and granulomatous meningoencephalomyelitis (GME) are common idiopathic inflammatory diseases of the central nervous system (CNS) in the dog. Several reports have contributed to clarify the clinical and histopathological features of canine idiopathic encephalitis. NME is characterized by nonsuppurative necrotizing lesions in the cerebral cortex, and is predisposed to small-sized dogs especially the Pug [27]. NLE is also characteristic of necrotizing demyelinating lesions in the white matter in very limited breeds including the Yorkshire Terrier [16, 18, 25, 27]. GME is characterized by focal or multifocal granulomatous lesions consisting of perivascular histiocytic infusion in the brain and cervical spinal cord [27].

On the other hand, the specific causes remain unclear among these diseases. To date, no causative infectious factor has been identified. Recently, researchers have been pointing out that these canine diseases have autoimmune pathogenesis. For example, Uchida et al. [29] and our previous works $[21,22,24,28]$ revealed the high prevalence of autoantibodies against glial fibrillary acidic protein (GFAP) in the cerebrospinal fluid (CSF) from dogs with

\footnotetext{
*Correspondence to: Matsuki, N., Department of Veterinary Clinical Pathobiology, Graduate School of Agricultural and Life Sciences, The University of Tokyo, 1-1-1 Yayoi, Bunkyoku, Tokyo 113-8657, Japan.

e-mail:amki@mail.ecc.u-tokyo.ac.jp
}

(C) 2012 The Japanese Society of Veterinary Science
NME. However, the anti-GFAP autoantibodies are not fully specific to NME [21], or do not directly attack the living astrocytes [23]. We have also shown the presence of IgGs targeting proteins other than GFAP in the CSF of NME cases [24].

In humans, several autoantibodies have been reported as the markers of CNS inflammatory disorders. For example, anti-aquaporin 4 antibodies in neuromyelitis optica [10], anti-N-methyl D-aspartate receptor (NMDAR) antibodies in NMDAR encephalitis [14], anti-glutamate receptor 3 antibodies in Rasmussen syndrome [20] and anti-transglutaminase (TG) antibodies in CNS celiac disease are currently known disease-specific autoantibodies [6]. In our preliminary study we surveyed these autoantibodies in the canine CSF and found a presence of anti-TG autoantibodies in the canine idiopathic encephalitis.

TGs are members of a family of $\mathrm{Ca}^{2+}$-dependant enzymes with principally protein-crosslinking activities [11, $13,15,19,26]$. Of the eight active TGs, TG2 is the most ubiquitously expressed in mammals including the brain [5, $15,17]$. Its role in the tissue homeostasis is variable and associations with pathological conditions such as fibrosis, atherosclerosis, neurodegenerative diseases, autoimmune diseases and cancer metastasis have been documented [2, $15,17]$. TG2 is also the major autoantigen in human celiac disease, a gluten-generated systemic disorder including the inflammatory encephalopathy $[6,8,9,11,12]$. In this study, we report the anti-TG2 antibodies in the CSF of canine idiopathic encephalitis. 


\section{MATERIALS AND METHODS}

Canine CSF samples: Samples of CSF were collected from 69 dogs being investigated for CNS diseases at the Veterinary Medical Center, the University of Tokyo between 2001 and 2010. The dogs were divided into four groups: 19 NME cases, 7 NLE cases, 11 GME cases, and 12 cases with other CNS diseases including 3 with canine distemper virus (CDV) encephalitis, 2 with CNS malignant histiocytosis, 2 with CNS lymphoma, 1 with glioma, and 4 with idiopathic epilepsy. All dogs were diagnosed by magnetic resonance imaging (MRI), routine CSF examinations and/or postmortem examinations. CSF was collected from the cisterna magna under general anesthesia, stored at $-30^{\circ} \mathrm{C}$ and thawed just prior to the analysis. CSF samples from clinically healthy dogs (9 Labrador Retrievers and 11 Beagles) were used as controls.

Immunohistochemistry: Autoantibodies in the CSFs were examined by an indirect fluorescence antibody test (IFA). A cryoblock of the cerebrum from a healthy female Beagle dog was kindly gifted from Professor Sasaki, Department of Veterinary Surgery, the University of Tokyo. Cryosections $(10 \mu \mathrm{m})$ were air-dried and fixed in cold acetone (Wako Pure Chemical, Osaka, Japan) for 5 min. Subsequently, the sections were washed with phosphate-buffered saline (PBS) for 5 min and blocked with normal goat serum (Wako) in PBS (1:100) for $30 \mathrm{~min}$. To demonstrate the presence of anti-TG2 antibodies, CSFs from dogs with NME were tested. For the preabsorption of anti-GFAP autoantibodies, the CSFs were incubated with purified bovine GFAP $(250 \mu \mathrm{g} / \mathrm{m} l$ CSF: Progen Biotechnik, Heidelberg, Germany) at room temperature for $1 \mathrm{hr}$. The sections were reacted with the NME-CSF with/ without the GFAP preabsorption at room temperature for $1 \mathrm{hr}$. After washes, the sections were labeled with FITCconjugated goat anti-dog $\operatorname{IgG}$ (1:400, Bethyl Laboratories, Montogomery, TX, U.S.A.) for $1 \mathrm{hr}$ at room temperature. Double-color staining was carried out to seek the colocalization of TG2 and antibodies in the NME-CSF. An anti-TG2 rabbit polyclonal IgG (1:50, Santa Cruz Biotechnology, Santa Cruz, CA, U.S.A.) was added to the NME-CSF preabsorbed with GFAP as indicated above, and incubated for $1 \mathrm{hr}$ at room temperature. After washes, the sections were labeled in green by FITC-conjugated goat anti-dog IgG, and also labeled by rhodamine-conjugated goat anti-rabbit IgG (1:100, Santa Cruz) for $1 \mathrm{hr}$ at room temperature. All the sections were washed with PBS and were mounted with Vectashield (Vector Labs, Burlingame, CA, U.S.A.) then examined by a fluorescent microscope (Optiphoto-2, Nikon, Tokyo, Japan) equipped with an ORCA-1394 image analyzing system (Hamamatsu Photonics, Hamamatsu, Japan).

Immunoblotting for anti-TG2 autoantibodies: A 0.5 $\mu \mathrm{g}$ of human recombinant TG2 (Immunodiagnostik AG, Bensheim, Germany) was subjected to sodium dodecyl sulfate-polyacrylamide gel electrophoresis (SDS-PAGE) on a $10 \%$ gel. The TG2 was transferred onto a nitrocellulose membrane (Hybond ECL, Amersham Pharmacia Biotech, Piscataway, NJ, U.S.A.). The membrane was blocked by 5\% skim milk (Becton Dickinson, Spards, MD, U.S.A.) in
Tris-buffered saline containing $0.1 \%$ Tween 20 (TBST) for $2 \mathrm{hr}$, and incubated with diluted CSFs (from 2 NME cases, 2 NLE cases and 2 GME cases; 1:100) for $1 \mathrm{hr}$. After washes, autoantibodies were reacted with horseradish peroxidaseconjugated goat anti-canine IgG (1:4,000, Bethyl) for $1 \mathrm{hr}$ and visualized by a chemiluminescence kit (ECL and Hyperfilm ECL, Amersham).

ELISA for anti-TG2 autoantibodies: Anti-TG2 IgG antibodies in the canine CSFs were measured by an ELISA. Briefly, 96-well microtiter plates (Sumitomo Bakelite, Tokyo, Japan) were coated with $0.2 \mu \mathrm{g} /$ well of human recombinant TG2 (Immunodiagnostik AG). The plates were blocked with PBS plus 3\% bovine serum albumin (BSA Fraction V ; Sigma-Aldrich, St. Louis, MO, U.S.A.) for 2 hr. After three washes with TBST, diluted CSF samples (1:20) were added $(50 \mu l /$ well $)$ in duplicate to corresponding wells and incubated at room temperature for $2 \mathrm{hr}$. After three washes with TBST, a $100 \mu l$ of horseradish peroxidaseconjugated goat anti-canine IgG (Bethyl) $(1: 2,000)$ was added to each well and incubated at room temperature for $1 \mathrm{hr}$. After washes, the plates were developed with $1 \mathrm{mg} /$ $\mathrm{m} l$ 2,2'-azinobis 3-ethylbenzothiazolin-6-sulfonic acid. The optical density at $405 \mathrm{~nm}$ was measured by a Model 680 plate reader (Bio-Rad Laboratories, Hercules, CA, U.S.A.).

Statistical analysis: ELISA data were evaluated using a Kruskal-Wallis test using GraphPad Prism 5 (La Jolla, CA, U.S.A.). Statistical significance was assigned at $P<0.05$.

\section{RESULTS}

Detection of anti-transglutaminase autoantibodies in the $C S F$ : To confirm the presence of CSF autoantigens against cerebral components, an IFA on the canine cerebral tissue was performed. Figure 1A shows strong immunoreactivity of the CSF from a NME dog to astrocytes. No immunoreactivity was observed in the CSF from a healthy control (Fig. 1B). After the preabsorption of the CSF with purified bovine GFAP, the immunoreactivity to astrocytes was still observed at a less extent (Fig. 1C). A double immunofluorescence assay using an anti-TG2 antibody revealed that the CSF reactivity was consistent with the localization of glial TG2 (Fig. 1D and 1E).

Immunoblot analysis for TG2 autoantibodies: Presence of TG2 autoantibodies in the CSF from dogs with NME, NLE and GME were detected by immunoblotting against human TG2 protein. As shown in Fig. 2, a common single band was detected at approximately $80 \mathrm{kDa}$ in the idiopathic encephalitis dogs, while no band was detected in the controls. Samples from miscellaneous diseases showed negative response to the IFA analysis and thereby were not conducted to the immunoblot analysis.

ELISA: The ELISA-OD values of anti-TG2 IgG in dogs with various CNS inflammatory diseases and healthy controls are shown in Fig. 3. The OD values of NME dogs were significantly higher than those of control dogs. Dogs with GME and NLE tended to show high values as compared to healthy controls. 


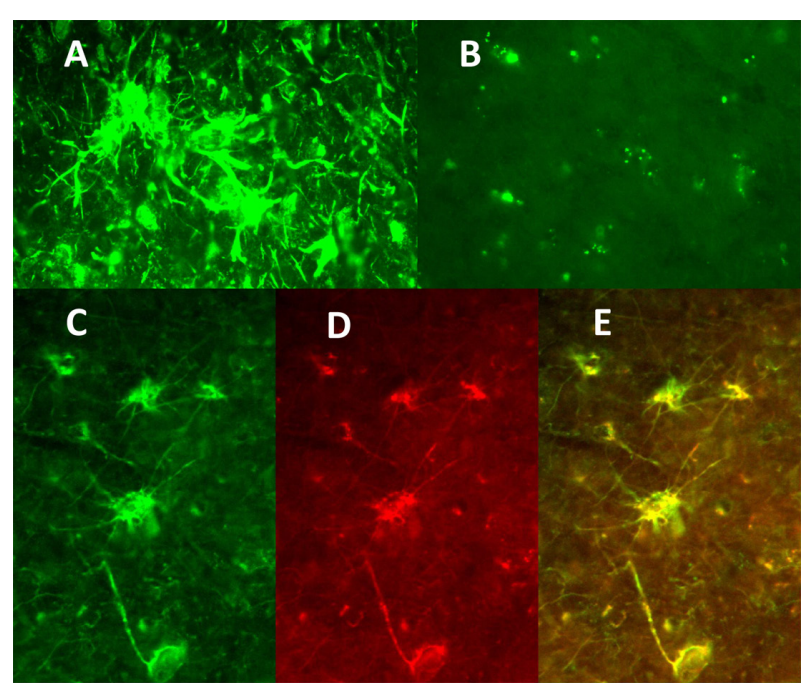

Fig. 1. Indirect fluorescent antibody test (IFA) on canine cerebral cryosections. Positive signals on astrocytes demonstrated by IgG in the CSF of canine necrotizing meningoencephalitis (A), IgG after absorption with purified bovine GFAP (C; green signal by FITC), anti-human TG2 polyclonal antibody (D; red signal by rhodamine), and common sites labeled by both antibodies (E; merged yellow signal). No signals were detected in the control (B).

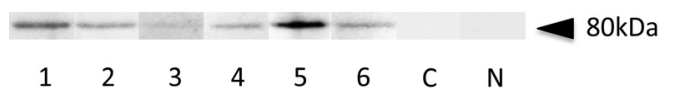

Fig. 2. Immunoblot against human recombinant TG2 protein with CSFs of canine idiopathic encephalitis. Necrotizing meningoencephalitis (NME; lanes 1 and 2), necrotizing leukoencephalitis (NLE; lanes 3 and 4), granulomatous meningoencephalomyelitis (GME; lanes 5 and 6), control CSF (C) and second antibody alone $(\mathrm{N})$. A common band at approximately $80 \mathrm{kDa}$ was detected in the canine idiopathic encephalitis cases.

\section{DISCUSSION}

Many studies on NME, NLE and GME revealed the predisposed breeds and the clinical courses of these diseases, however, the exact cause and etiology remains undetermined in each disease [27]. We previously reported the high correlation of CSF anti-GFAP antibodies with NME cases $[21,22,24,28]$, and sought to clarify the pathogenic antigens from the perspective of humoral immunity. We also demonstrated that the anti-GFAP autoantibodies in NME dogs do not directly damage astrocytes in vitro [23], and also referred to the possibility of other autoantigens [24]. Hence, in this study we aimed to identify novel autoantigens in the canine CSF.

In order to preclude anti-GFAP antibodies from the CSF of NME dogs, we preabsorbed the CSF with bovine GFAP. The preabsorbed CSF still showed the reactivity to canine

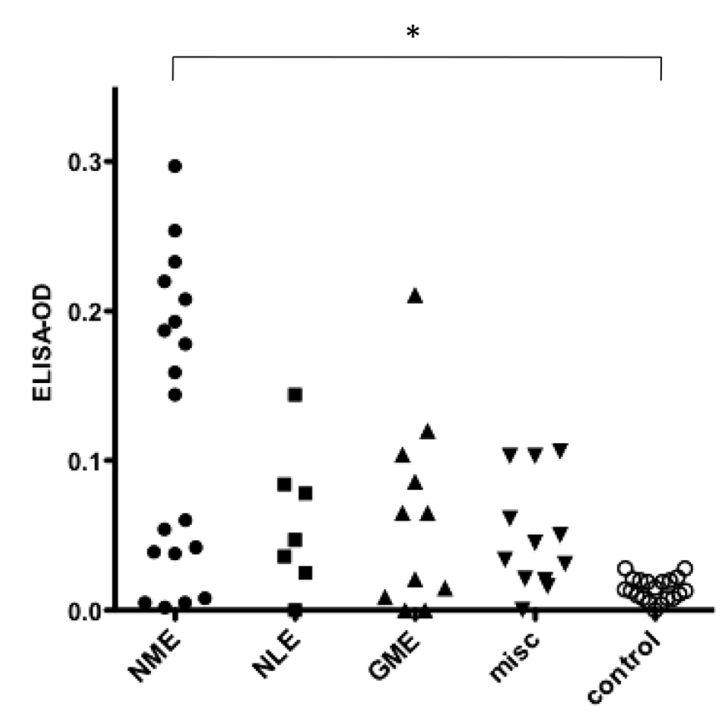

Fig. 3. Autoantibodies against human recombinant TG2 protein in the CSFs of dogs with necrotizing meningoencephalitis (NME), necrotizing leukoencephalitis (NLE), granulomatous meningoencephalomyelitis (GME), miscellaneous CNS diseases (misc) and controls. Each symbol represents the mean optical density of duplicate assays; ${ }^{*} P<0.05$.

astrocytes. The double-immunofluorescent staining using anti-human TG2 IgG demonstrated that the CSF autoantibody binds to canine TG2. In fact, our immunoblot analysis against human-recombinant TG2 protein revealed the clear reaction of NME-CSF to human-TG2 of approximately 80 $\mathrm{kDa}$. Unexpectedly, the immunoblot analysis showed the presence of anti-TG2 antibodies in the CSF of NLE and GME cases. Our ELISA procedure for the CSF anti-TG2 IgG showed high prevalence of anti-TG2 IgG in canine idiopathic encephalitis, while NME cases showed relatively higher titers of anti-TG2 IgG than NLE and GME cases. As shown in our IFA data, TG2 is mainly distributed in astrocytes in the canine brain. TG2 is known to play roles to keep the integrity of blood-brain-barrier [1, 11, 12]. We speculate that the higher titers of anti-TG2 autoantibody in NME cases may reflect the damage of blood-brain-barrier or loss of other astrocytic functions caused by necrotizing inflammation.

The majority of NME cases assessed in the present study were positive for CSF anti-GFAP autoantibodies in our previous reports. We found no significant relationship between anti-GFAP and anti-TG2 autoantibody titers in these NME cases (data not shown). On the other hand, almost all of NLE and GME cases in the present study were negative for anti-GFAP autoantibodies, thus, we currently speculate that anti-GFAP and anti-TG2 autoantibodies were independently generated by different causes.

TG2 is widely distributed throughout the human tissue including the brain $[5,15,17]$. In human celiac disease, IgG and/or IgA antibodies against TG2 are known to be serum 
hallmarks [6]. Celiac disease is a systemic disorder strongly associated with human leukocyte antigen (HLA) haplotypes and a part of celiac patients show chronic neurological manifestations especially cerebellar ataxia (CNS celiac disease) [6]. Reports have shown that the sera from celiac disease patients with anti-TG antibodies induced transient ataxia in mice $[3,4]$ and mitochondrial-dependent apoptosis in cultured neuronal cells [7], suggesting the neurotoxicity of anti-TG2 antibodies.

In human CNS celiac disease, the histopathological findings of the brain are characterized by lymphocytic infiltration and perivascular cuffing especially in the white matter of cerebrum and cerebellum, associated with cerebellar atrophy $[6,12]$. The distribution of brain lesions and clinical symptoms in human CNS celiac disease are quite different from those of canine NME, NLE or GME. However, considering the contribution of TG2 in the CNS functions $[1,2,5,11-13,15]$, we can speculate that the inflammatory characteristic of canine idiopathic encephalitis may be provoked by the autoimmunity to TG2.

By the lack of specificity to NME, NLE or GME, we cannot utilize the canine CSF anti-TG2 autoantibodies as a disease marker, however, we can expect the autoantibodies as an essential candidate to consider the undiscovered pathogenesis of canine idiopathic encephalitis. It affords a clue that these diseases might share a common mechanism in the initiation or progression of inflammation. Further study is needed to elucidate whether the anti-TG2 autoantibodies are produced intrathecally or peripherally, as well as whether the autoantibodies are the cause or result of diseases.

ACKNOWLEDGMENT. This work was supported in part by a Grant-in-Aid for Scientific Research from the Ministry of Education, Culture, Sports, Science and Technology of Japan (Number 20380169).

\section{REFERENCES}

1. Aeschlimann, D. and Paulsson, M. 1991. Cross-linking of laminin-nidogen complexes by tissue transglutaminase. A novel mechanism for basement membrane stabilization. J. Biol. Chem. 266: 15308-15317. [Medline]

2. Aeschlimann, D. and Thomazy, V. 2000. Protein crosslinking in assembly and remodelling of extracellular matrices: the role of transglutaminases. Connect. Tissue Res. 41: 1-27. [Medline] [CrossRef]

3. Boscolo, S., Lorenzon, A., Sblattero, D., Florian, F., Stebel, M., Marzari, R., Not, T., Aeschlimann, D., Ventura, A., Hadjivassiliou, M. and Tongiorgi, E. 2010. Anti transglutaminase antibodies cause ataxia in mice. PLoS ONE 5: e9698. [Medline] [CrossRef]

4. Boscolo, S., Sarich, A., Lorenzon, A., Passoni, M., Rui, V., Stebel, M., Sblattero, D., Marzari, R., Hadjivassiliou, M. and Tongiorgi, E. 2007. Gluten ataxia. Ann. N.Y. Acad. Sci. 1107: 319-328. [Medline] [CrossRef]

5. Caccamo, D., Campisi, A., Curro, M., Li Volti, G., Vanella, A. and Ientile, R. 2004. Excitotoxic and post-ischemic neurodegeneration: involvement of transglutaminases. Amino Acids 27: 373-379. [Medline] [CrossRef]
6. Caja, S., Mäki, M., Kaukinen, K. and Lindfors, K. 2011. Antibodies in celiac disease: implications beyond diagnostics. Cell. Mol. Immunol. 8: 103-109. [Medline] [CrossRef]

7. Cervio, E., Volta, U., Verri, M., Boschi, F., Pastoris, O., Granito, A., Barbara, G., Parisi, C., Felicani, C., Tonini, M. and De Giorgio, R. 2007. Sera of patients with celiac disease and neurologic disorders evoke a mitochondrial-dependent apoptosis in vitro. Gastroenterology 133: 195-206. [Medline] [CrossRef]

8. Dieterich, W., Ehnis, T., Bauer, M., Donner, P., Volta, U., Riecken, E. O. and Schuppan, D. 1997. Identification of tissue transglutaminase as the autoantigen of celiac disease. Nat. Med. 3: 797-801. [Medline] [CrossRef]

9. Dieterich, W., Laag, E., Schopper, H., Volta, U., Ferguson, A., Gillett, H., Riecken, E. O. and Schuppan, D. 1998. Autoantibodies to tissue transglutaminase as predictors of celiac disease. Gastroenterology 115: 1317-1321. [Medline] [CrossRef]

10. Fazio, R., Radaelli, M. and Furlan, R. 2011. Neuromyelitis optica: concepts in evolution. J. Neuroimmunol. 231: 100-104. [Medline] [CrossRef]

11. Hadjivassiliou, M., Aeschlimann, P., Strigun, A., Sanders, D. S., Woodroofe, N. and Aeschlimann, D. 2008. Autoantibodies in gluten ataxia recognize a novel neuronal transglutaminase. Ann. Neurol. 64: 332-343. [Medline] [CrossRef]

12. Hadjivassiliou, M., Sanders, D. S., Grünewald, R. A., Woodroofe, N., Boscolo, S. and Aeschlimann, D. 2010. Gluten sensitivity: from gut to brain. Lancet Neurol. 9: 318-330. [Medline] [CrossRef]

13. Iismaa, S. E., Mearns, B. M., Lorand, L. and Graham, R. M. 2009. Transglutaminases and disease: lessons from genetically engineered mouse models and inherited disorders. Physiol. Rev. 89: 991-1023. [Medline] [CrossRef]

14. Irani, S. R. and Vincent, A. 2011. Autoimmune encephalitis-new awareness, challenging questions. Discov. Med. 11: 449-458. [Medline]

15. Jeitner, T. M., Pinto, J. T., Krasnikov, B. F., Horswill, M. and Cooper, A. J. L. 2009. Transglutaminases and neurodegeneration. J. Neurochem. 109: 160-166. [Medline] [CrossRef]

16. Kuwamura, M., Adachi, T., Yamate, J., Kotani, T., Ohashi, F. and Summers, B. A. 2002. Necrotising encephalitis in the Yorkshire terrier: a case report and literature review. J. Small Anim. Pract. 43: 459-463. [Medline] [CrossRef]

17. Lesort, M., Tucholski, J., Miller, M. L. and Johnson, G. V. 2000. Tissue transglutaminase: a possible role in neurodegenerative diseases. Prog. Neurobiol. 61: 439-463. [Medline] [CrossRef]

18. Lezmi, S., Toussaint, Y., Prata, D., Lejeune, T., Ferreira-Neves, P., Rakotovao, F., Fontaine, J. J., Marchal, T. and Cordonnier, N. 2007. Severe necrotizing encephalitis in a Yorkshire terrier: topographic and immunohistochemical study. J. Vet. Med. A Physiol. Pathol. Clin. Med. 54: 186-190. [Medline] [CrossRef]

19. Lorand, L. and Graham, R. M. 2003. Transglutaminases: crosslinking enzymes with pleiotropic functions. Nat. Rev. Mol. Cell Biol. 4: 140-156. [Medline] [CrossRef]

20. Mastrangelo, M., Mariani, R. and Menichella, A. 2010. Eponym: Rasmussen syndrome. Eur. J. Pediatr. 169: 919-924. [Medline] [CrossRef]

21. Matsuki, N., Fujiwara, K., Tamahara, S., Uchida, K., Matsunaga, S., Nakayama, H., Doi, K., Ogawa, H. and Ono, K. 2004. Prevalence of autoantibody in cerebrospinal fluids from dogs with various CNS diseases. J. Vet. Med. Sci. 66: 295-297. [Medline] [CrossRef] 
22. Matsuki, N., Takahashi, M., Yaegashi, M., Tamahara, S. and Ono, K. 2009. Serial examinations of anti-GFAP autoantibodies in cerebrospinal fluids in canine necrotizing meningoencephalitis. J. Vet. Med. Sci. 71: 99-100. [Medline] [CrossRef]

23. Pham, N. T., Matsuki, N., Shibuya, M., Tamahara, S. and Ono, K. 2008. Impaired expression of excitatory amino acid transporter 2 (EAAT2) and glutamate homeostasis in canine necrotizing meningoencephalitis. J. Vet. Med. Sci. 70: 1071-1075. [Medline] [CrossRef]

24. Shibuya, M., Matsuki, N., Fujiwara, K., Imajoh-Ohmi, S., Fukuda, H., Pham, N. T., Tamahara, S. and Ono, K. 2007. Autoantibodies against glial fibrillary acidic protein (GFAP) in cerebrospinal fluids from Pug dogs with necrotizing meningoencephalitis. J. Vet. Med. Sci. 69: 241-245. [Medline] [CrossRef]

25. Spitzbarth, I., Schenk, H. C., Tipold, A. and Beineke, A. 2010. Immunohistochemical characterization of inflammatory and glial responses in a case of necrotizing leucoencephalitis in a French bulldog. J. Comp. Pathol. 142: 235-241. [Medline] [CrossRef]

26. Stamnaes, J., Dorum, S., Fleckenstein, B., Aeschlimann, D. and Sollid, L. M. 2010. Gluten T cell epitope targeting by TG3 and TG6; implications for dermatitis herpetiformis and gluten ataxia. Amino Acids 39: 1183-1191. [Medline] [CrossRef]

27. Talarico, L. R. and Schatzberg, S. J. 2010. Idiopathic granulomatous and necrotising inflammatory disorders of the canine central nervous system: a review and future perspectives. $J$. Small Anim. Pract. 51: 138-149. [Medline] [CrossRef]

28. Toda, Y., Matsuki, N., Shibuya, M., Fujioka, I., Tamahara, S. and Ono, K. 2007. Glial fibrillary acidic protein (GFAP) and anti-GFAP autoantibody in canine necrotising meningoencephalitis. Vet. Rec. 161: 261-264. [Medline] [CrossRef]

29. Uchida, K., Hasegawa, T., Ikeda, M., Yamaguchi, R. and Tateyama, S. 1999. Detection of an autoantibody from Pug dogs with necrotizing encephalitis (Pug dog encephalitis). Vet. Pathol. 36: 301-307. [Medline] [CrossRef] 